**TITLE**

ASP Conference Series, Vol. **VOLUME***, **YEAR OF PUBLICATION**

**NAMES OF EDITORS**

\title{
Maps of the millimetre sky from the BOOMERanG experiment
}

P. de Bernardis ${ }^{1}$, P.A.R. Ade ${ }^{2}$, A. Balbi ${ }^{3}$, J.J. Bock ${ }^{4}$, J.R. Bond ${ }^{5}$, J. Borrill ${ }^{6}$, A. Boscaleri ${ }^{7}$, P. Cabella ${ }^{3}$, C.R. Contaldi ${ }^{5}$, B.P. Crill ${ }^{8,9}$, G. De Gasperis ${ }^{3}$, A. De-Oliveira Costa $^{10}$, G. De Troia ${ }^{1}$, K. Ganga ${ }^{11}$, M. Giacometti ${ }^{1}$, E. Hivon ${ }^{11}$, V.V. Hristov ${ }^{8}$, T. Kisner ${ }^{12}$, A. Iacoangeli ${ }^{1}$, A.H. Jaffe ${ }^{13}$, W.C. Jones ${ }^{8}$, A.E. Lange ${ }^{8}$, S. Masi ${ }^{1}$, P. Mason ${ }^{8}$, P.D. Mauskopf ${ }^{2}$, C. MacTavish ${ }^{14}$, A. Melchiorri ${ }^{1}$, T. Montroy ${ }^{11}$, F. Nati $^{1}$, P. Natoli ${ }^{3}$, C.B. Netterfield ${ }^{14}$, E. Pascale ${ }^{7,14}$, F. Piacentini ${ }^{1}$, D. Pogosyan ${ }^{15}$, G. Polenta ${ }^{1}$, S. Prunet ${ }^{16}$, G. Romeo ${ }^{17}$, S. Ricciardi ${ }^{1}$, J.E. Ruhl ${ }^{12}$, M. Tegmark ${ }^{10}$, N. Vittorio ${ }^{3}$

1 Dipartimento di Fisica, Universita' La Sapienza, Roma, Italy

2 Dept. of Physics and Astronomy, Cardiff University, Wales, UK

3 Dipartimento di Fisica, Universita' di Tor Vergata, Roma, Italy

4 Jet Propulsion Laboratory, Pasadena, CA, USA

5 CITA, University of Toronto, Canada

${ }^{6}$ NERSC, LBNL, Berkeley, CA, USA

7 IFAC-CNR, Firenze, Italy

8 California Institute of Technology, Pasadena, CA, USA

9 CSU Dominguez Hills, Carson, CA, USA

10 Phys. Dept. University of Pennsylvanya, Philadelphia, PA, USA

11 IPAC, CalTech, Pasadena, CA, USA

12 Physics Department, CWRU, Cleveland, OH, USA

13 Astrophysics Group, Imperial College, London, UK

14 Depts. of Physics and Astronomy, University of Toronto, Canada

15 Physics Dept., University of Alberta, Alberta, Canada

16 Institut d'Astrophysique, Paris, France

17 Istituto Nazionale di Geofisica, Roma, Italy

Abstract. In the 1998-99 flight, BOOMERanG has produced maps of $\sim 4 \%$ of the sky at high Galactic latitudes, at frequencies of 90, 150, 240 and $410 \mathrm{GHz}$, with resolution $\gtrsim 10^{\prime}$. The faint structure of the Cosmic Microwave Background at horizon and sub-horizon scales is evident in these maps. These maps compare well to the maps recently obtained at lower frequencies by the WMAP experiment. Here we compare the amplitude and morphology of the structures observed in the two sets of maps. We also outline the polarization sensitive version of BOOMERanG, which was flown early this year to measure the linear polarization of the microwave sky at 150, 240 and $350 \mathrm{GHz}$. 


\section{Introduction}

BOOMERanG is balloon-borne microwave telescope, sensitive at 90, 150, 240 and $410 \mathrm{GHz}$, with a resolution of $\sim 10^{\prime}$. The instrument was equipped with very sensitive spider-web bolometers (Mauskopf et al. 1997). The image of the sky is obtained by slowly scanning the full payload in azimuth $\left( \pm 30^{\circ}\right)$ at constant elevation. The scan center constantly tracks the azimuth of the lowest Galactic foreground region, situated in the southern hemisphere, in the Horologium constellation. Every day of the flight the instrument obtains a fully cross-linked map of about $45^{\circ} \times 30^{\circ}$ of the sky. The instrument was flown in a long duration circum-Antarctic flight from Dec.28, 1998 to Jan.8, 1999, and has been described in Piacentini et al. (2002) and in Crill et al. (2003). The main target of the experiment was the detection of anisotropy in the Cosmic Microwave Background. The maps produced by the experiment have been published in de Bernardis et al. (2000), Masi et al. (2001), Netterfield et al. (2002), Ruhl et al. (2003). Due to the limited size and to $1 / \mathrm{f}$ noise, these maps do not contain information for angular scales $\gg 5^{\circ}$ and have been filtered accordingly. The maps are calibrated to $\sim 10 \%$ in gain and to $\sim 10 \%$ in beam FWHM. In the 150 $\mathrm{GHz}$ map, the signal is well above the noise, and maps taken at different scan speeds and in different locations are perfectly consistent, demonstrating the low level of systematic effects. The level of the noise is of the order of $50 \mu \mathrm{K}$ per $7^{\prime}$ pixel and of $20 \mu \mathrm{K}$ per $28^{\prime}$ pixel. At 90,150 and $240 \mathrm{GHz}$ the rms signal has the spectrum of CMB anisotropy, and does not fit any reasonable spectrum of foreground emission. The temperature fluctuations detected in the high latitude part of the $150 \mathrm{GHz}$ map are remarkably gaussian (Polenta et al. 2002, De Troia et al. 2003). The angular power spectrum of the $150 \mathrm{GHz}$ map in the multipole range $50<\ell<1000$ has been computed in Netterfield et al. (2002) and Ruhl et al. (2003). Three peaks have been detected in the power spectrum, at multipoles $\ell \sim 210,540,845$ (de Bernardis et al., 2002). Interstellar Dust contamination of the $150 \mathrm{GHz}$ power spectrum has been shown to be less than 1\% (Masi et al. 2001). These results fit the scenario of acoustic oscillations of the primeval plasma at horizon and subhorizon scales (Sunyaev and Zeldovich 1970, Peebles and Yu 1970). In the framework of adiabatic inflationary structure formation the cosmological parameters have been estimated from the measured power spectrum (Lange et al. 2001, Netterfield et al., 2002, de Bernardis et al. 2002, Ruhl et al. 2003). The three cosmological parameters best constrained by the BOOMERanG data are the curvature parameter $\Omega=1.03 \pm 0.05$ (the universe is nearly flat), the spectral index of the primordial density perturbations $n_{s}=1.02 \pm 0.07$ (nearly scale invariant), the physical density of baryons $\Omega_{b} h^{2}=0.023 \pm 0.003$ (consistent with Big Bang Nucleosynthesis).

Here we compare the BOOMERanG maps to the maps recently obtained at similar frequency and resolution by the WMAP satellite (Bennett et al., 2003). Working from the advantage L2 point of the Sun-Earth system, in its first year of operation WMAP has produced full sky maps of the microwave sky at $22,32,41,60,94 \mathrm{GHz}$, with resolution of the order of $15^{\prime}-30^{\prime}$ and noise of $\sim 35 \mu K$ per pixel in 28 ' pixels. The maps are precisely calibrated (better than 1\%). The power spectra obtained from these maps are fully consistent with the CMB power spectrum measured by BOOMERanG at $150 \mathrm{GHz}$, and with the adiabatic inflationary scenario: they allow the precise determination 
of most of the cosmological parameters; these estimates improve the precision of the BOOMERanG ones by a factor 2-3 for the parameters mentioned above; the full-sky coverage and polarization sensitivity allows the determination of previously poorly constrained parameters, like the reionization optical depth (Spergel et al. 2003).

The comparison of the BOOMERanG and WMAP maps is carried out with three targets:

- Compare independent maps of the CMB with similar angular resolution, and confirm the detection of primordial structures in both experiments;

- Improve the BOOMERanG calibration using the precise calibration of WMAP, and demonstrate that $1 \%$ gain calibration is possible for the new, polarizationsensitive BOOMERanG-B2K survey;

- Infer the level and properties of foregorund contamination in forthcoming deep surveys of the CMB.

\section{BOOMERanG vs WMAP}

In fig. 1 we compare the maps of BOOMERanG at 90, 150, $240 \mathrm{GHz}$ to the maps of WMAP at 41, 60, $94 \mathrm{GHz}$ in the same high latitude region. The maps are Healpix (Gorski et al. 1998) representations with npix $=512$ (7' per pixel). The region selected has the best coverage in the BOOMERanG $150 \mathrm{GHz}$ channels. $\mathrm{A} \sim 5^{\circ}$ high-pass has been applied to the WMAP maps to easy the comparison to the BOOMERanG maps, which are intrinsically insensitive to large angular scales.

The morphological agreement of the structures detected is evident. Significant differences are in the level of the noise and in the presence of important contamination by AGNs in the lower frequency channels. Once these sources are masked, the pixel-pixel correlation between the maps is quite good.

A quantitative analysis must take into account all important differences between the two datasets:

- The beams of BOOMERanG and WMAP are different. The BOOMERanG beam at $150 \mathrm{GHz}$ is closely fit by a 11' gaussian down to $2 \%$ of the axial gain, while at lower levels is better fit by a 13' gaussian. The WMAP beam at $94 \mathrm{GHz}$ is fit by a 13' gaussian down to $5 \%$ of the axial gain, and has wide "shoulders" at lower levels. This difference is difficult to treat in pixel space, while its treatment is relatively simple in multipoles space. An example of this can be found in Abroe et al. (2003), comparing MAXIMA and WMAP maps of the CMB.

- The BOOMERanG maps do not include structures larger than $10^{\circ}$, while WMAP maps are accurate at all scales. Once again, this is easier to account for in multipoles space.

- In this pixelization, the noise of the $150 \mathrm{GHz}$ BOOMERanG map is around $50 \mu \mathrm{K}$ per pixel, while the noise of the $94 \mathrm{GHz}$ WMAP map is around $180 \mu \mathrm{K}$ per pixel.

A full analysis taking into account all these details is described in Hivon et al. (2003): the main result is that the calibration of BOOMERanG-98 at 150 $\mathrm{GHz}$ is found to be consistent with the precise calibration of WMAP within 5\%. 

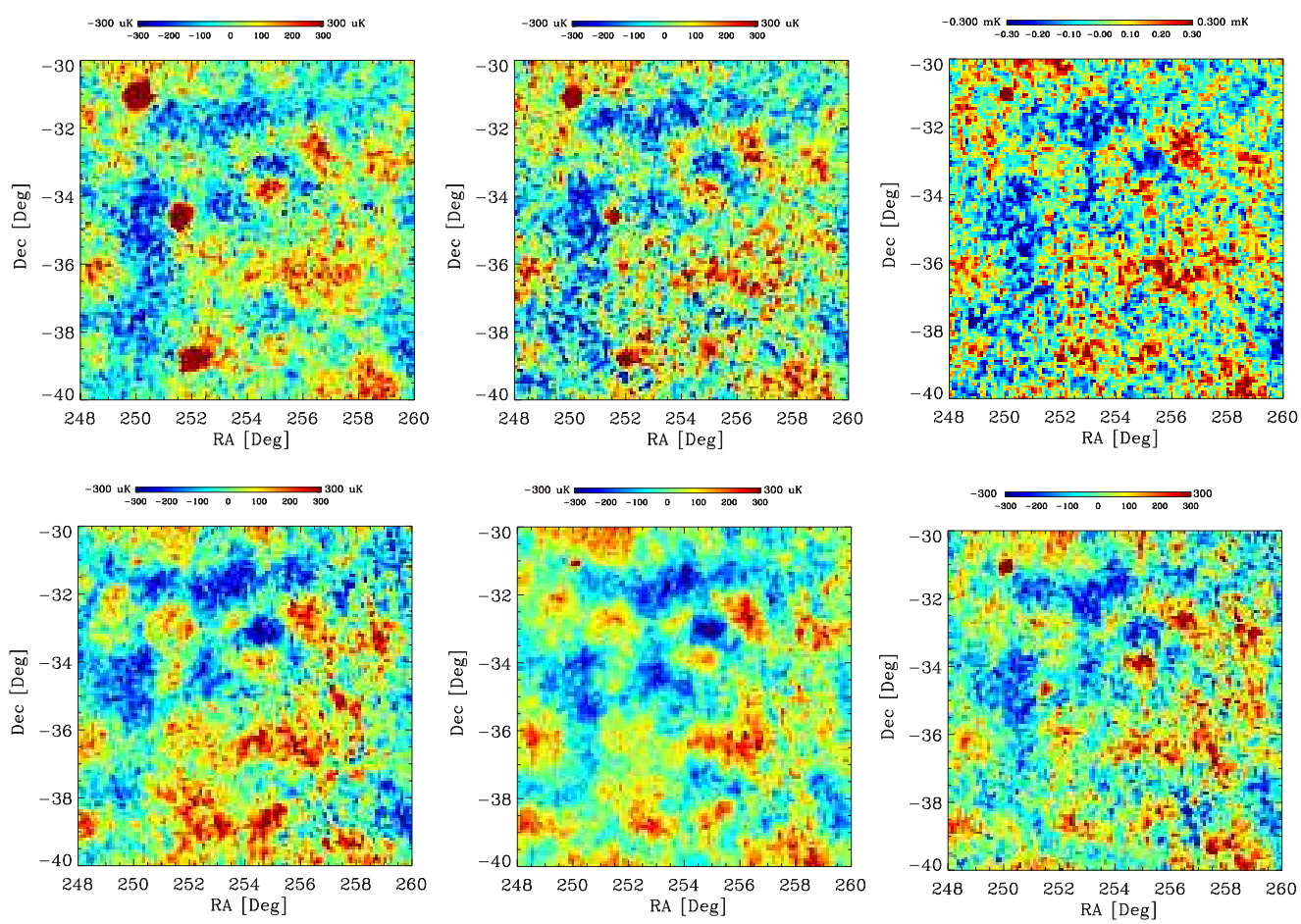

Figure 1. A sample $\sim 10^{\circ} \times 10^{\circ}$ region at high galactic latitudes as seen by WMAP (top row, 41, 61, $94 \mathrm{GHz}$ left to right) and by BOOMERanG-98 (bottom row, 220, 150, $90 \mathrm{GHz}$ left to right). The grey scale is in thermodynamic temperature units for the CMB $(-300 \mu K<\Delta T<300 \mu K)$. The coordinates are Galactic $\left(248^{\circ}<\ell<\right.$ $\left.260^{\circ},-40^{\circ}<b<-30^{\circ}\right)$. The pixel size is $7^{\prime}$. It is evident the very good agreement of the $\mathrm{CMB}$ maps obtained by the two experiments (compare the two $\sim 90 \mathrm{GHz}$ maps on the right). Note the decrease of the equivalent brightness of the three AGN sources (evident at $41 \mathrm{GHz}$ ) with increasing frequency, and the very low noise of the $150 \mathrm{GHz}$ map by BOOMERanG (center panel in bottom row). The WMAP maps have been filtered to remove structures larger than $5^{\circ}$, which cannot be detected by BOOMERanG. 


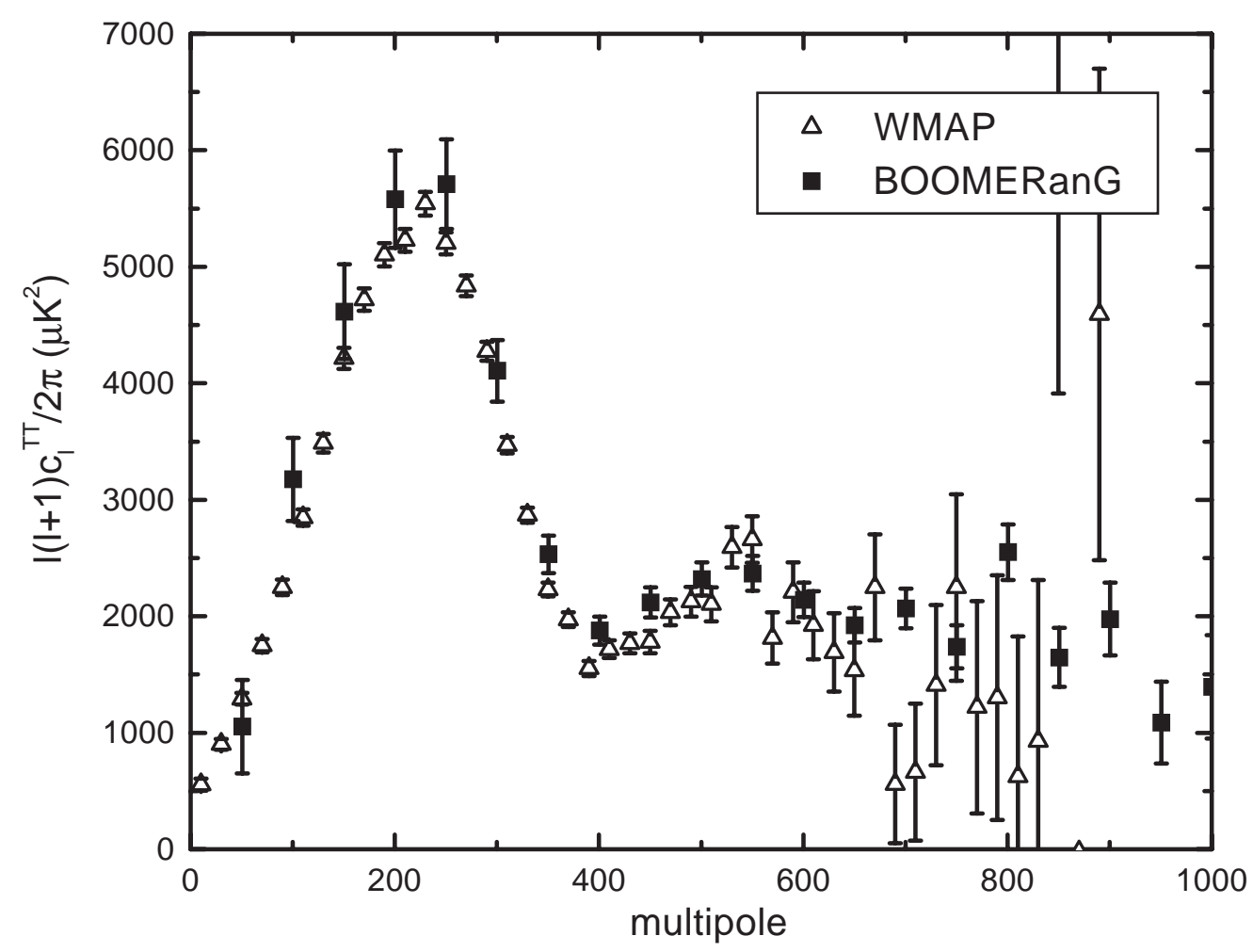

Figure 2. Comparison of the angular power spectra of the CMB measured by WMAP (open triangles, $\Delta \ell=20$ bins) and by BOOMERanG (squares, $\Delta \ell=50$ bins). The error bars represent random errors only.

Moreover, $<1 \%$ calibration of BOOMERanG is found to be reachable with this correlation method.

Given the close consistency of the maps of BOOMERanG and WMAP, the consistency of the angular power spectra measured by the two experiments is almost a trivial consequence. In fig.2 we compare the angular power spectra detected by the two instruments. The gain calibration error $(<1 \%$ for WMAP and $\sim 10 \%$ for BOOMERanG) is not included in the error bars, which account for random errors only. It is evident that the WMAP experiment has much higher sensitivity than BOOMERanG at multipoles $\lesssim 450$ : the WMAP measurement in this range is limited by cosmic variance, and the Power Spectrum can be considered definitive. In the region of the second peak the two experiments have similar sensitivity, while in the region of the third peak the BOOMERanG experiment has better sensitivity due to the smaller beam and the lower noise per pixel. For the determination of the cosmological parameters $\Omega, \Omega_{b} h^{2}, n_{s}$, in the adiabatic inflationary scenario, WMAP takes advantage of the accurate calibration, while the BOOMERanG is still competitive because of the wider multipoles coverage, including the third peak of the spectrum. These results give only an idea of what can be expected from Planck-HFI (Lamarre et al. 2003), the satellite instrument developed to fully exploit the capabilities of cryogenic bolometers. This will work for about two years from the same deep space 


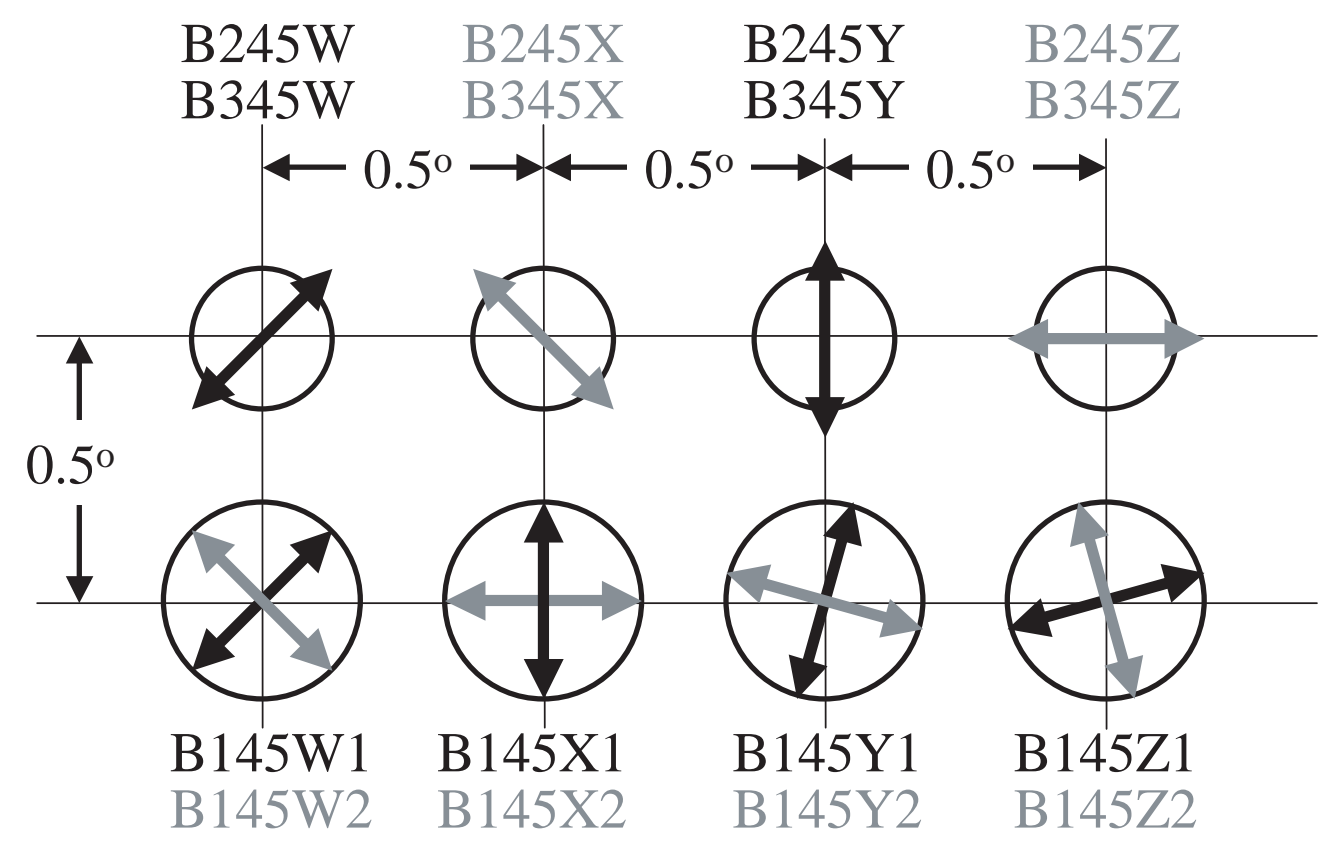

Figure 3. The focal plane of B2K. The azimuth (scan) direction is horizontal. The 8 pixels host two bolometers each, labeled by the frequency in $\mathrm{GHz}$ and by the principal axis of the polarization sensitivity pattern. The FWHM is $\sim 9^{\prime}$ for the $145 \mathrm{GHz}$ PSBs; is $\sim 6^{\prime}$ for the 245 $\mathrm{GHz}$ and $345 \mathrm{GHz}$ channels.

location as WMAP, using bolometric detectors at $0.1 \mathrm{~K}$, even more sensitive than the BOOMERanG ones.

\section{The polarization-sensitive BOOMERanG: B2K}

After the 1998-99 flight, BOOMERanG has been recovered and upgraded. Additional attitude sensors have been implemented (a day-time star camera developed in Toronto and a pointed star sensor developed in Rome), and the focal plane has been rebuild to accomodate polarization sensitive bolometers (PSB) developed in JPL/Caltech (Jones et al. 2003). The new focal plane is sketched in fig.3. It has 8 pixels in two rows. The distance between pixels projected in the sky is $0.5^{\circ}$ both in azimuth and in elevation. Each pixel of the bottom row is a $145 \mathrm{GHz}$ PSB with two independent bolometers sensing the two orthogonal polarization directions (Jones et al. 2003); each pixel on the top row senses a single polarization direction in two frequencies (240 and $350 \mathrm{GHz}$ ). The 150 GHz beam is $9^{\prime}$ FWHM, while the 240 and $350 \mathrm{GHz}$ beams are $\sim 6^{\prime}$ FWHM. More details on the instrument can be found in Montroy et al. (2003). The experiment has been designed to measure the $\mathrm{E}$ modes (gradient) in the CMB polarization pattern. This has been detected only by the DASI experiment, but the sensitivity was not enough to constrain cosmological models more than with anisotropy data. WMAP has published a detection of the TE cross correlation 

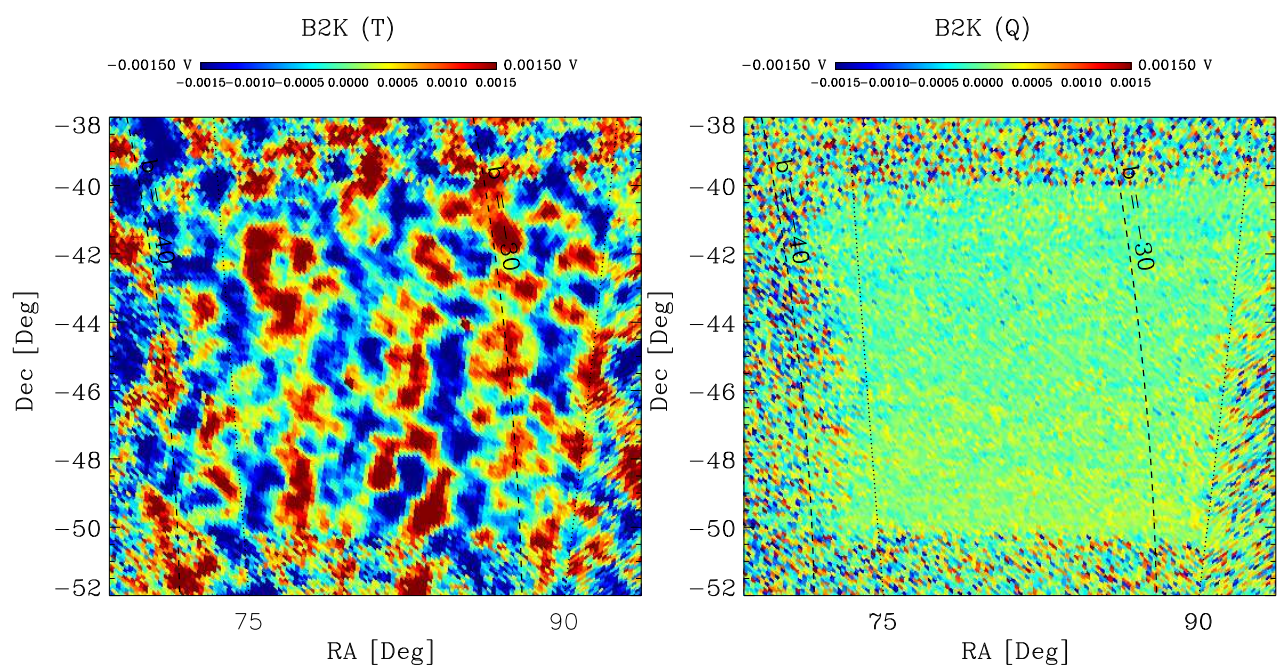

Figure 4. Left: Preliminary CMB anisotropy map in the region with deeper integration surveyed during the B2K flight. The signals from the 8 PSB bolometers sensitive at $145 \mathrm{GHz}$ have been averaged to obtain the brightness map. The map has been obtained from the IGLS optimal code (Natoli et al. 2001). The brightness units are not absolutely calibrated and the pointing solution is preliminary. Right: Map of Stokes parameter Q obtained from the 8 PSB bolometers, in the same units as the anisotropy map. Linear polarization of the CMB can be extracted from the noise by means of power spectrum analysis. 
from the first year of operation, consistent with the adiabatic model inferred by the anisotropy measurement. B2K should be able to measure the TE and EE power spectra at frequencies higher than the DASI and WMAP ones (30 to $94 \mathrm{GHz}$ ), thus facing different polarized foregrounds and nicely complementing them. The B2K payload has been flown from the McMurdo base on Jan.7, 2003, for a total of 11 days of operation in the stratosphere. In fig.4 we plot preliminary maps from B2K obtained from the PSBs at $145 \mathrm{GHz}$. We plan to re-fly B2K with an upgraded focal plane, to go after the polarized foreground from cirrus dust and AGNs. This information is essential for all the planned B-modes experiments (e.g. BICEP, Dome-C etc.) and is very difficult to measure from ground. The BOOMERanG optics can host an array of $\gtrsim 100 \mathrm{PSB}$ at $\gtrsim 350$ $\mathrm{GHz}$, providing a deep, high resolution survey of polarized foreground emission at frequencies close to the ones used for CMB polarization research.

The BOOMERanG experiment is supported in Italy by Agenzia Spaziale Italiana, Programma Nazionale Ricerche in Antartide, Universita' di Roma La Sapienza; by PPARC in the UK, by NASA, NSF OPP and NERSC in the U.S.A., and by CIAR and NSERC in Canada.

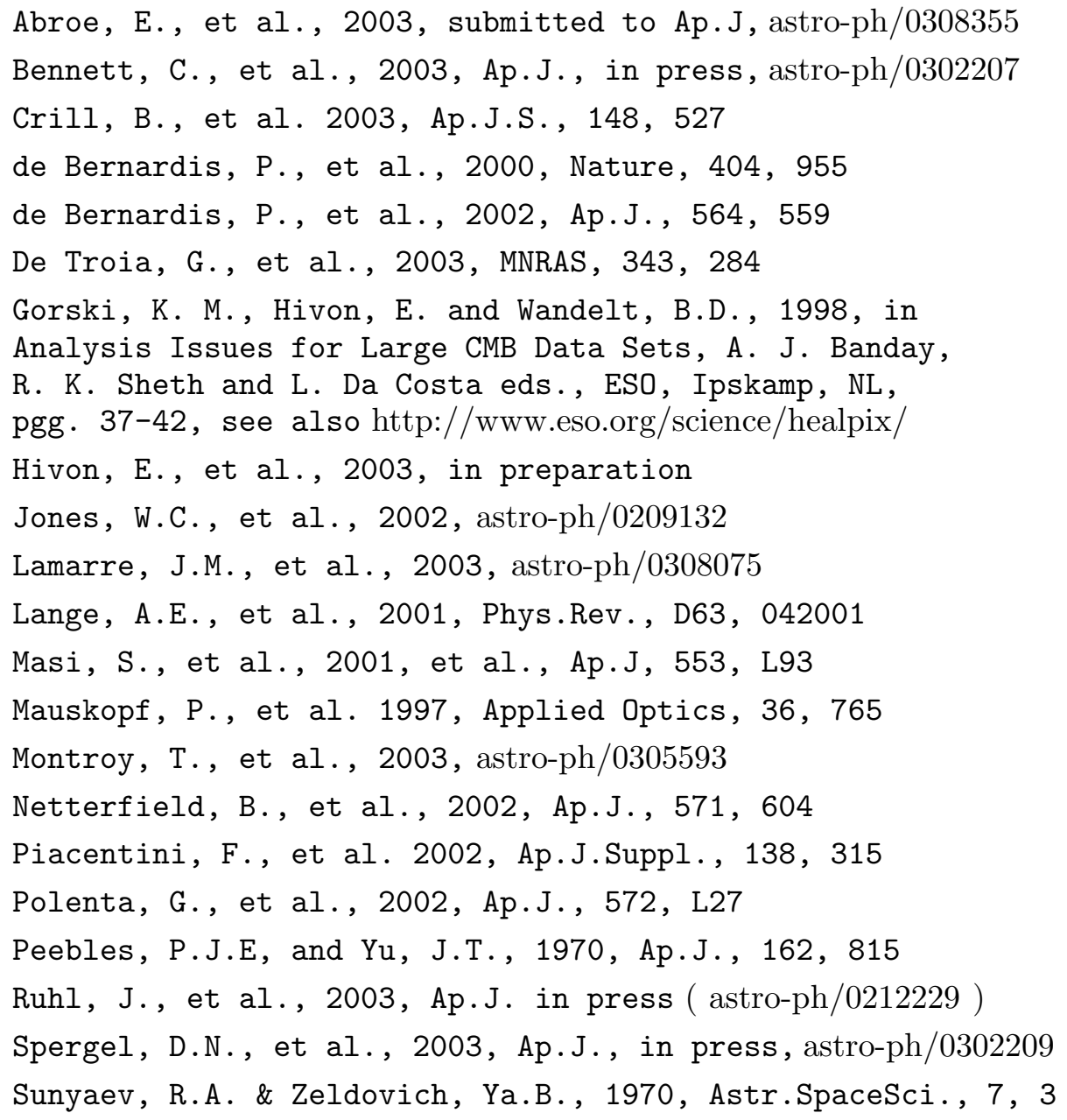

